\title{
The research based on private cloud computing information interaction model
}

\author{
Meng Zhang ${ }^{1, a}$, Zheng Liang ${ }^{1, b}$, Zhen Li $^{3 \mathrm{c}}$ \\ 1 Shandong Medical College Ji'nan,Shandong,P.R.China, 250002 \\ aredboyzm@163.com, 'blulu19660617@163.com, 'lizhen2338@126.com
}

Keywords: private cloud computing, Information interactive model, MNVPN, Layer of service, Layer of resource

\begin{abstract}
The author analyses the current problems of campus network, make a research about the construction of university campus network, through the resource integration of campus network, and then introduce a information interaction model based on private cloud computing information interaction model, which includes the layer of user, layer of service and layer of resource. It can make the user to visit the mass information through on access internet, and enjoy fastest, highest quality information transmission service anytime and anywhere.
\end{abstract}

\section{Introduction}

Cloud computing is a further production of the computer science and Internet technology, also is the important strategic technology and means to the future information industry innovation. As the appearance of cloud computing which is the new network core technology computing platform, Internet access from software to service as the center of "cloud" time. Followed, education information resources construction which is an important part of education informatization in our country also will meet new challenges.

From April 2003, as early start project of "quality project", the ministry of education started high quality courses construction in every university across the country. High quality courses refer to the excellent courses which has distinctive and first-class teaching level. High quality courses construction should reflect modern education thoughts, conform to the scientific, advanced and universal law education teaching, with distinctive features, and can properly use modern teaching technology, method and means, teaching effect is remarkable, with the function of demonstration and radiation[1].

Under the promotion of ministry of education, the construction of high quality courses in colleges and universities has achieved fruitful results. By the end of March 2013, total named 4410 national high quality courses, including 2513 courses in regular colleges, 1045 courses in vocational colleges, 209 courses in network education, 143 courses in military academies (including armed police)[2], every provinces and universities also attaches great importance to the construction of high quality courses, construction and selected a large number of high quality courses. The fundamental purpose of high quality courses construction of is to improve the teaching quality, promoting quality education resources sharing service, however, the high quality courses in the process of information transmission often involve huge amounts of data storage and processing, due to the limit of public network access bandwidth, cannot meet the requirements of the fast data exchange, result in the high quality courses resources application effect is not ideal, course access and interaction problem is the most prominent. If using the cloud platform to provide various services, will bring new vitality of the efficient use of high quality courses resources.

The author analysised and studied the problem of a university high quality courses resources outside page view is not good, found the main reason is the university's network not underutilized, limited the information transmission, and slow response time and so on. According to the characteristics of the campus network, therefore, this article puts forward an information interaction model architecture based on private cloud computing, to solve the limited information transmission problem, in order to improve network resource utilization. 


\section{The Cloud Platform Deployment and Network Topology}

\section{The Way of Cloud Deployment Platform}

Using cloud computing technology can entities a number of relatively low cost computing to a super integrated computing and resource environment through the network, unified management and scheduling computing resources when through a network, constitute a computational resources pool, and with the aid of infrastructure services (IaaS), platform services (PaaS), software services (SaaS) and other advanced service mode provide services according to the need of customers, realize computing resources, storage resources, data resources, information resources, knowledge resources, expert resources, equipment resources co-construction and sharing.

Cloud platform can be deployed three different types[3]: Public cloud, Private cloud and Hybrid cloud.

Public cloud deployed in an open environment, provide the services to the user and the system which has the ability to access, its biggest feature is the user access through the Internet and use public cloud services, but don't have the cloud and cloud management.

Private cloud is deployed in a specific environment (network closed or service scope closed) of a cloud computing system. The system's system boundary is clear, only provide the services to the specified range customers.

Hybrid cloud is a combination of public and private cloud. It usually consists of two or more clouds (public clouds and private clouds), through standardized technical or professional binding together, realize the portability between the cloud data and application, solve the load balancing of cloud burst between cloud.

The author according to the actual situation of a university information resource access private cloud deployment, the main reason is the following three aspects:

(1) The effective control of safety and service quality. Create the cloud infrastructure software and hardware resources within the local area network firewall, users can control the deployed application on the infrastructure, so provide the effective control of data security and service quality.

(2) To improve the information transmission speed, saving the cost of hardware and software investment. Because of the current local area network (LAN) internal exchange rate has reached megabytes even gigabit, if using private cloud can not only make full use of network resources to improve the speed of information transmission, but also can save the server and software input costs.

(3) The high efficient and accurate service. Private cloud can provide a variety of hardware and software resource sharing service, but also let users quick access to a large number of resources, then get high efficient and accurate service.

Of course, with the upgrading of network infrastructure and perfecting laws, private cloud can be gradually opened to the Internet, transition into hybrid cloud, eventually provide high quality public cloud services to many users.

\section{Network Topology}

The establishment of private cloud network based on the Intranet, the network topology is shown in figure 1.

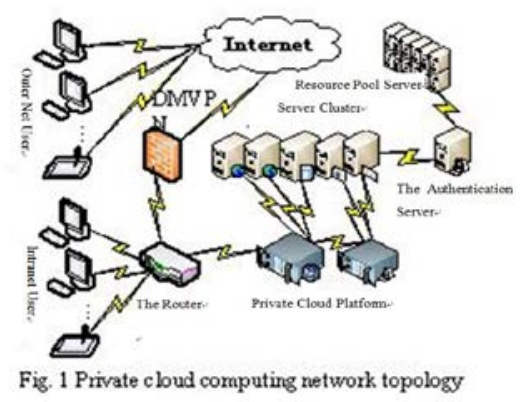

The key problem of network construction is need to though DMVPN [2] (Dyncmic Multipoint Virtual Private Network) connect different regions resources into a logically unified internal 
network, then adopting the firewall technology isolation Internet and Private cloud area. Within the private cloud terminal access link bandwidth of at least 100 MBPS, trunk link bandwidth can be more than 1 GBPS. All the equipment and terminal supports the transmission control protocol/Internet protocol (TCP/IP), including routers, switches, minicomputers, and various servers [4].

\section{Private Cloud Information Interaction Model}

Private cloud computing information interaction model, as shown in figure 2. The model used three layers of structure: the user layer, service layer and resource layer. Every layers contact through the interface, each layer provides services to its upper layer, it easy to scale horizontally between the layer, extend the functionality; it Easy to longitudinal extension and variation between the layer. Each layer can also separate provide different functions and levels of service to outside, at the same time, three layer combination can provide service for the user. The information transmission process is mainly divided into four stages: authorization, upload, transfer, and the end phase.

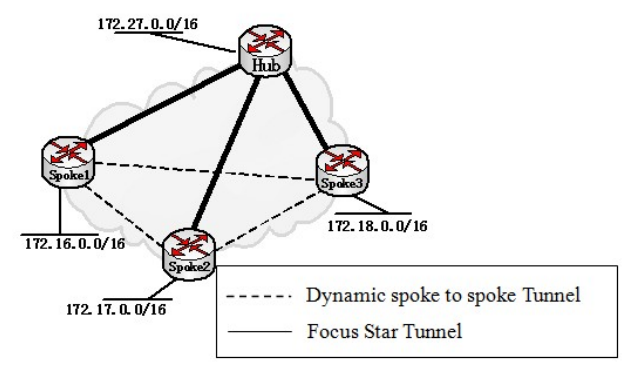

Fig. 2 IP was transferred in DMVPN tunnel

\section{The Structure Design of Three Layers}

In the structure design of three layers, the user layer to the end user provide a variety of powerful functions, and flexible using software services, users can get the application via browser interface such as a WEB, and each kind of application software can be used by multiple users at the same time[5]. This section focuses on the service layer and resource layer design.

\section{The Service Layer Design}

Service layer between resource layer and user layer, all the software in this layer has generality and reusability, and has perfect elasticity and high availability automatically. By using the layer provides users develop and test environment, after the user complete development and testing, it only need to pass the packed application task to the user layer, and then by parsing the metadata information configuration for the application, make the application layer can be normal use its rely service platform.

Service layer Consists of four modules, the application software, the virtual machine, the virtual access, and the calculation, of which, the calculation module belong to the public module of service layer and resource layer. Each module adopts a service-oriented architecture SOA [4] pattern design. Through this architectural design can meet the users needed information directly through the browser mapped to the service layer of related components, at the same time can also block the difference of platform and data sources, more effectively fusion the multiple resources.

1) Application Software Module

In the virtual environment of service layer, all the applications in preconfigured virtual machine images provided by the end user or provided to end users, including resource distribution automation, users self-service portal technology, etc.

2) Virtual Machine Module

Virtual machine module includes virtual server and virtual network. Network virtual can integrated different function of network into a whole virtual, on the basis of share physical network, 
set up the logic paths which can be transmitted across a wide area network or isolated independent, as shown in figure 3.

Virtual server is use the hypervisor abstracts from the physical server virtual computing environment, conversion lots of servers which can make full use in data center into a seamless resources pool, to a great extent, simplifies intertwined together and produce complexity of the servers, storage and network, as shown in figure 4.

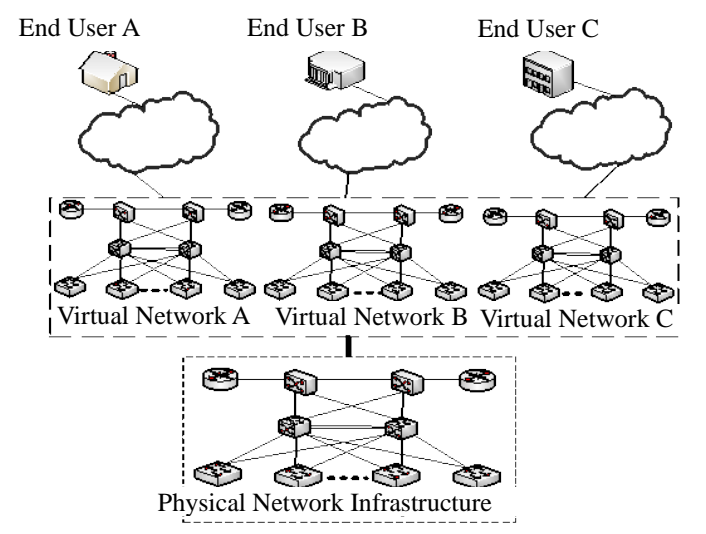

Fig. 3 Network

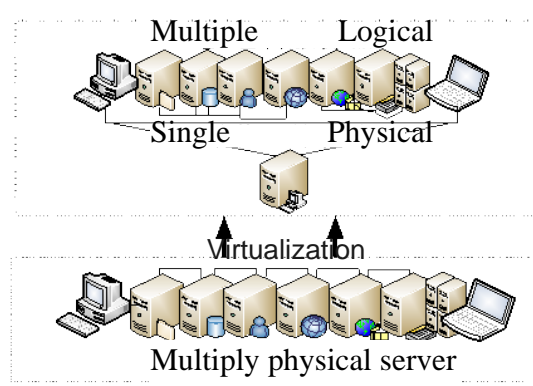

Fig. 4 The virtual server consolidation

3) Virtual Access Module

The port parameters configuration information of virtual access module as shown in figure 5 . This module is to build a virtual access layer switches, implement multiple extensions between lots of virtual machine to the network interface, solve the point to point scalability problems from the physical interface to virtual interface.

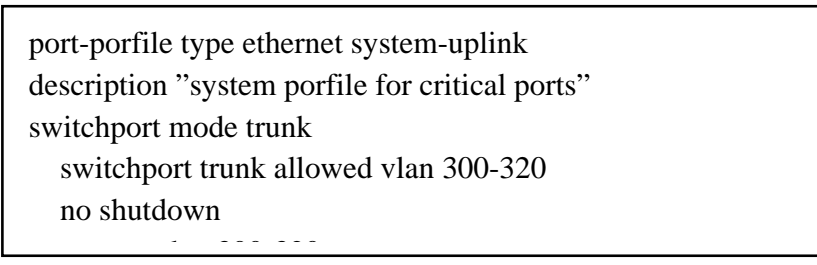

Fig. 5 Parameters of the virtual machine interface

\section{Resources Layer Design}

Resources layer is a collection of virtualization hardware resources and related management function, its architecture is shown in figure 6. Resource layer mainly includes server module calculation sub-module, storage module, optical module and wan module. Based on this architecture model can be dynamically allocated the user needs, implement internal process automation and resource management, optimization, then providing dynamic and flexible resources service to the outside, achieve rapid transmission of information.

In the resource layer structure, first to make sure the differences between different brand and types of hardware of the servers, storage and network equipment, through the storage virtualization technology shielding, then through the service virtualization technology to integrate all the hardware, building a resource pool, and standardize management of the unified logic and interface. Eventually, transfer all the information through the DMVPN between inside or outside.

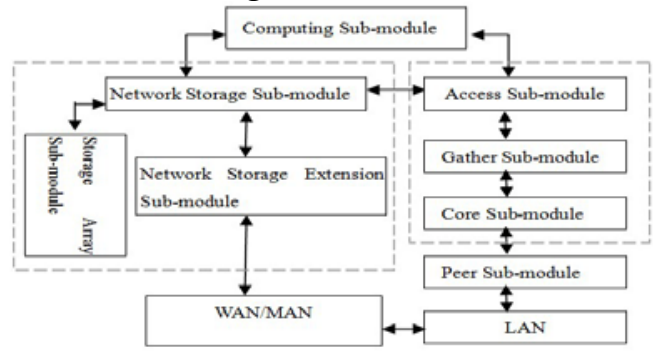

Fig. 6 The architecture of resource layer

\subsubsection{Calculation Sub-module}


Calculation sub-module is the core of the private cloud computing, using IBM servers, mainly complete virtualization and file configuration management. Calculation sub-module adopted technology mainly includes: memory expansion and file management.

(1) memory expansion: calculation sub-module adopted the standard rate of double synchronous dynamic random access memory type 3 DDR3 memory[6], changing memory by logical structure, realize the CPU memory extension control, calculation sub-module on every four DIMM dual-in-line storage module is mapped to the CPU memory controller in a single logical DIMM, make it become a four times before a single storage capacity. Solved spread service of the jumps server, saved large amounts of memory space for the virtual software runs multiple operating systems.

(2) unified file management: calculation sub-module management program belongs to the embedded device management software, through the intuitive graphical user interface management, as a single logical entity to manage private cloud system, put all kinds of identity components information in the configuration file, created a unified management area, make the service configuration file and the template realized the management based on the strategy and the role. Simplify the servers move configuration between data center, make the disaster recovery site and calculation sub-module directly connected, timely backup the information through calculating sub-module.

\subsubsection{Storage Module}

Storage module is mainly used to provide data storage resources for private cloud computing, consists of the network storage sub-module, the storage array sub-module, network storage extension sub-module. Network storage sub-module realizes the storage array and interconnection between private cloud computing platform, connect the storage unit which have been separated from the private cloud platform processing unit again. Storage array sub-module responsible for separated the storage capacity from calculation sub-module and then integration, maximize efficiency as much as possible. network storage extension sub-module can connected multiple geographically distributed data centers into a virtual cluster data through DMVPN, realize the connection of the local area network (LAN) and metropolitan area network and wide area network within the scope of Internet data center, so provide more quality services for terminal resource layer, ensure the continuity of the business, when the accident happens, it can timely recovery the disaster. The solution of the module as shown in figure 7.



\subsubsection{Fiber Module}

Fig. 7 The expansion plan of storage area network

Fiber module responsible for the data transmission between the different private cloud module, including access sub-module, core sub-module, gather sub-module. Access layer sub-module implementation services for a variety of network to deploy in order to meet the needs of network storage and application, let the storage and application of information flow using the unified I/O for network transmission, and combined fiber and Ethernet. Gather sub-module bind the network flow which from the access layer, to ensure the rational use of physical resources, realize the firewall, load balancing and other virtual services. Core sub-module use of virtual routing and forwarding function of this layer switches the third layer partition from wan extension to the data center, to ensure the layer 3 network transmission and infrastructure to mutual isolation, solved the resources for large-scale deployment of high scalability.

\subsubsection{Peer Module}

This module located in the center data of LAN and on the edge of the WAN, realized the operation of all kinds of local area network and metropolitan area network, specially designed for 
the peer application such as GRE tunnel, IPSec Settings, by LAN or the Internet using multi-point dynamic virtual private network (DMVPN) provide services to remote branches or the end user.

4.2.5 Dynamic Multipoint Virtual Private Network (DMVPN)

DMVPN is a kind of network can support a fully connected network without the need for network of all the connection path in advance, in this network, each branch all support the limited tunnel between the centre routing, at the same time support any connection of the activity current state and other branches, even a branch will not be able to support more branch tunnel, it can still through the branch tunnel to transmit data to the center.

IPSec element encapsulation mechanism, using DMVPN may lead to cannot predict in advance routing which based on a particular branch of the tunnel. So in the Internet key exchange IKE[7] security association adopted an address mask (0.0.0.0 0.0.0.0), allows all the IP address initialization to a new IPSec session.

\section{Conclusion}

In the private cloud computing environment which has built campus network (shown in figure 1), startup experiment test of the private cloud computing server. Organized students about 1000 using the computer in computer room at the same time, through the campus network boutique online learning course, the whole system can correct parsing, scheduling and execution

The test results shows that this paper proposed information interaction model based on private cloud computing, could make full use of network resources, made information transmission smoothly and fast response time, solved the problem of limited information transmission, raised the utilization ratio of network resources.

\section{Reference:}

[1] Takayuki Yamada. Remarks on tracking method of neural network weight change for adaptive type neural network feed forward feedback controller[J]. Artificial Life and Robotics, 2008,131:

[2] Sam Johnston. Cloud Computing Type Public Cloud, Hybrid Cloud, Private Cloud [EB/OL].(2009-3)[2011-5]http://www.circleid.com/posts/20090306_cloud_computing_types_p ublic_hybrid_private/

[3] Dynamic Multipoint Virtual Private Network [EB/OL].(2013-3)[2013-4] http://en.wikipedia.org/wiki/Dynamic_Multipoint_Virtual_Private_Network

[4] HUA Xiang. Research on Framework of Private Cloud in Visual Simulation [J]. JOURNAL OF SYSTEM SIMULATION, 2011, (23) : 1652-1655.

[5] C.L.Liu, M.Nakagawa ,Precise Candidate Selection for Large Character Set Recognition by Confidence Evaluation, IEEE Trans Pattern Analysis and Machine Intelligence, Vol.22,no.6,PP.636 - 642,2000.

[6] Vitaly Surazhsky, Tatiana Surazhsky, Danil Kirsanov et al. Fast Exact and Approximate Geodesics on Meshes: Proceedings of ACM SIGGRAPH 2005 papers,2005 [C]. Los Angeles: ACM Press, 2005: 553-560.

[7] Jones M W, Satherley R. Voxelisation: modeling for volume graphics: Proceedings of the 2000 Conference on Vision Modeling and Visualization 2000[C]. Berlin: Aka GmbH , 2000, 319-326. 